University of Nebraska - Lincoln

DigitalCommons@University of Nebraska - Lincoln

$12-20-2004$

\title{
Magnetoelectronics with magnetoelectrics
}

\author{
Christian Binek \\ University of Nebraska-Lincoln, cbinek@unl.edu \\ Bernard Doudin \\ University of Nebraska-Lincoln, bernard.doudin@ipcms.unistra.fr
}

Follow this and additional works at: https://digitalcommons.unl.edu/physicsbinek

Part of the Physics Commons

Binek, Christian and Doudin, Bernard, "Magnetoelectronics with magnetoelectrics" (2004). Christian Binek Publications. 28.

https://digitalcommons.unl.edu/physicsbinek/28

This Article is brought to you for free and open access by the Research Papers in Physics and Astronomy at DigitalCommons@University of Nebraska - Lincoln. It has been accepted for inclusion in Christian Binek Publications by an authorized administrator of DigitalCommons@University of Nebraska - Lincoln. 


\title{
LETTER TO THE EDITOR
}

\section{Magnetoelectronics with magnetoelectrics}

\author{
Ch Binek and B Doudin \\ Department of Physics and Astronomy and the Center for Materials Research and \\ Analysis, University of Nebraska-Lincoln, Lincoln, NE 68588-0111, USA
}

Received 20 October 2004, in final form 8 December 2004; Published 20 December 2004

\begin{abstract}
Magnetoelectric films are proposed as key components for spintronic applications. The net magnetic moment created by an electric field in a magnetoelectric thin film influences the magnetization state of a neighbouring ferromagnetic layer through exchange coupling. Pure electrical control of magnetic configurations of giant magnetoresistance spin valves and tunnelling magnetoresistance elements is therefore achievable. Estimates based on documented magnetoelectric tensor values show that exchange fields reaching $100 \mathrm{mT}$ can be obtained. We propose a mechanism alternative to current-induced magnetization switching, providing access to a wide range of device impedance values and opening the possibility of simple logic functions.
\end{abstract}

The emerging field of spintronics is already successful for applications in magnetic readheads and sensors. They involve giant magnetoresistance (GMR) [1-3] and tunnel magnetoresistance (TMR) effects [4], which provide a change of resistance related to a modification of the magnetic configuration between two neighbouring ferromagnetic films. Evolution beyond passive magnetoelectronic components is envisioned in the next generation of spintronics devices, which should combine memory and logical functions and promises to set new standards in future information technology [5]. There has been growing interest in studying a direct method for magnetization reversal involving spin transfer from a spin-polarized current injected into the device. This effect has been theoretically predicted by Slonczewski [6] and Berger [7], and has been experimentally confirmed by several groups [8-12].Experiments and theory agree on the necessity of applying significant current densities (larger than $10^{11} \mathrm{~A} \mathrm{~m}^{-2}$ ) for switching the orientation of the magnetic nanoparticle. Scaling down of the device area makes spin-transfer an attractive alternative to stray magnetic field techniques for samples of size below $100 \mathrm{~nm} \times 100 \mathrm{~nm}$. However, the technical difficulties involved in making reliably such small structures, the necessity to apply large currents and avoid heating of the samples, and the intrinsic low sample resistance (of the order of a few ohms) are limiting the practical use for GMR devices. Applications in TMR devices are hindered by the large current density enforced in a very thin insulator, and the few reports on TMR systems are not conclusive [13, 14]. 
We propose device architectures combining a GMR or TMR device with a magnetoelectric (ME) film, where the electric field is used as alternative means for controlling the magnetic configuration of spintronics devices. ME properties were originally predicted by Curie, concurrently to the discovery of ferromagnetism [15]. In an ME material, an electric field $\mathrm{E}$ induces a magnetic moment $\mathrm{M}=\alpha \mathrm{E}$, where $\alpha$ is the tensor of ME susceptibility. Landau and Lifschitz clarified the necessary crystalline symmetry conditions [16], which are the absence of temporal and spatial inversion, but invariance with respect to the combination of both operations. Breaking time and spatial inversion symmetry can be realized by magnetic order and electric polarization, respectively. The prototypical ME example is the antiferromagnetic $\mathrm{Cr}_{2} \mathrm{O}_{3}[17,18]$, where electric polarization and magnetization are field induced. Evidence of $\mathrm{ME}$ properties has also been found for several other materials: $\mathrm{GaFeO}_{3}, \mathrm{Ni}_{3} \mathrm{~B}_{7} \mathrm{O}_{13} \mathrm{I}, \mathrm{LiMnPO}_{4}$ or $\mathrm{Y}_{3} \mathrm{Fe}_{5} \mathrm{O}_{12}[19]$.

Recently, major attention has been paid to the ME effect in magnetic ferroelectrics where polarization spontaneously occurs [20]. Among these, the multiferroic systems exhibit a coexistence of spontaneous ferromagnetic and ferroelectric order, and the coupling between magnetization and polarization gives rise to a pronounced ME response [21]. It is needless to say that a variety of spectacular applications, in particular in the field of non-volatile data storage, can be expected if ultimately reversal of one order parameter is achieved by applying the conjugate field of its coexisting counterpart. Recently, there have been promising demonstrations of cross coupling involving polarization reversal by magnetic fields in $\mathrm{TbMn}_{2} \mathrm{O}_{5}$ and electric field stimulated ferromagnetic order in the hexagonal $\mathrm{HoMnO}_{3}[22,23]$. Epitaxially grown films of $\mathrm{BiFeO}_{3}$ provide an attractive alternative to bulk multiferroic phases due to enhanced polarization and magnetization caused by constraints of heteroepitaxial growth [24].

In this letter we focus on spintronic applications which take explicit advantage of the antiferromagnetic properties of ME material. Antiferromagnetic order is only slightly affected by moderate external magnetic fields, but provides control of the magnetic configuration of spintronic devices through strong exchange coupling to adjacent ferromagnetic films. Electric fields tune the coupling via the ME effect and provide a novel degree of freedom, allowing the implementation of simple logical functions.

In the simplest microscopic description of the prototypical $\mathrm{ME}$ compound $\mathrm{Cr}_{2} \mathrm{O}_{3}$ [25], $\mathrm{Cr}^{3+}$ ions located on different sublattices are shifted by the field to non-equivalent positions and experience distinct strength of the crystal field. This changes the g-tensor and the single ion anisotropy in a non-equivalent way, and modifies the exchange integrals between the ions. The magnetic moment created by an electric field remains small. For $\mathrm{Cr}_{2} \mathrm{O}_{3}$ with typical applied electric fields of the order of $10^{5} \mathrm{~V} \mathrm{~m}^{-1}$ on a millimetre-sized sample, the magnetic moment is only of the order of $10^{-5} \mu_{\mathrm{B}}$ per $\mathrm{Cr}$ atom. However, if we apply electric fields reaching dielectric breakdown values of thin films $\left(10^{9} \mathrm{~V} \mathrm{~m}^{-1}\right)$, the extrapolation of the linear $\mathrm{M}(\mathrm{E})$ behaviour predicts a magnetic moment of several percent of $\mu_{\mathrm{B}}$ per atom. If an ME layer is adjacent to a ferromagnetic film, exchange coupling allows a shift $\mu_{0} \mathrm{H}_{\mathrm{e}}$ of the ferromagnet hysteresis, with magnitudes becoming relevant for applications as spin valves. Estimates by Hochstrat et al [26] indicate that an exchange field of $0.2 \mathrm{mT}$ is expected for an applied electric field of $10^{5} \mathrm{~V} \mathrm{~m}^{-1}$. Linear extrapolation predicts values of the order of $100 \mathrm{mT}$ if electric fields approaching dielectric breakdown values are applied.

Experimental data providing indications of $\mathrm{ME}$ influence on magnetoresistance properties have been reported in $\mathrm{CrO}_{2} / \mathrm{Cr}_{2} \mathrm{O}_{3} / \mathrm{CrO}_{2}$ junctions, where the $\mathrm{Cr}_{2} \mathrm{O}_{3}$ film is naturally growing between two $\mathrm{CrO}_{2}$ crystallites. These results were originally presented in the framework of current-induced switching models, questioning however the small current density occurring in these devices [27]. Figure 3 shows that the conductance versus voltage of these junctions exhibits hysteretic and asymmetry behaviour at temperatures higher 


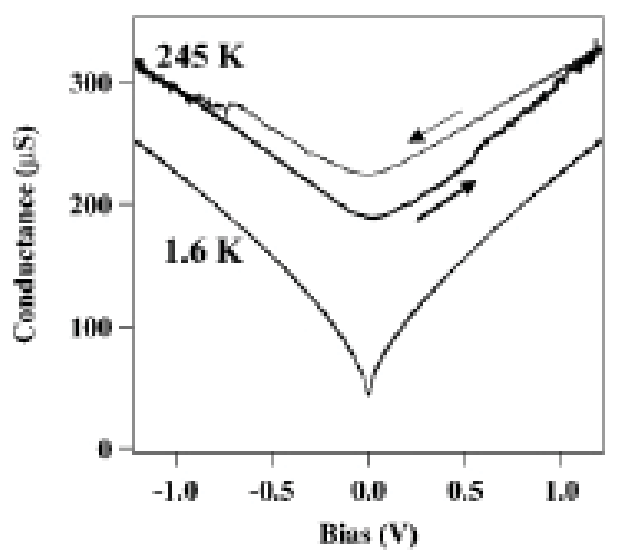

Figure 1. Conductance versus bias voltage for a $\mathrm{CrO}_{2} / \mathrm{Cr}_{2} \mathrm{O}_{3} / \mathrm{CrO}_{2}$ junction. Asymmetry and hysteresis of the curve are absent at temperatures typically below $20 \mathrm{~K}$, corresponding to the negligible magnetoelectric properties of $\mathrm{Cr}_{2} \mathrm{O}_{3}$.

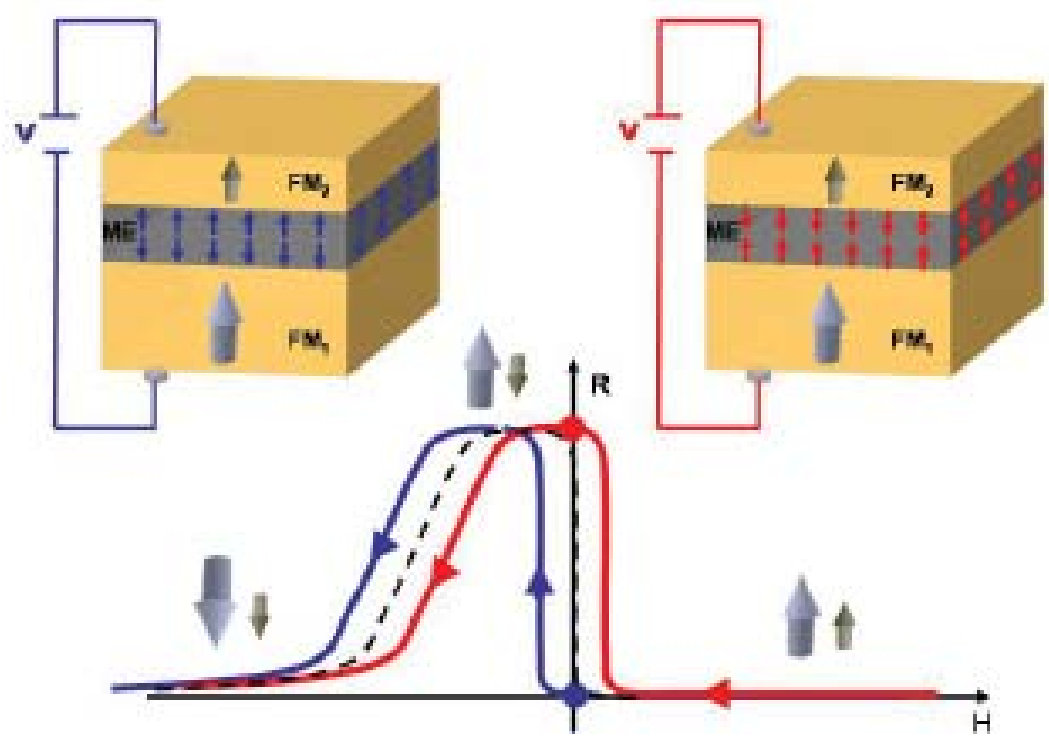

Figure 2. Schematics of the magnetoresistance curve of a TMR device involving an ME film as a tunnel barrier. Half-hysteresis curves are shown, after saturation at positive field values. The arrows denote the magnetization directions, with the bottom layer FM1 being harder (or pinned) than the top one FM2. The dashed curve is the expected TMR behaviour. The change of voltage polarity changes the direction of the net magnetization of the ME layer, adding an exchange bias magnetic field to the resistance curve. The two colours indicate shifting of half-hysteresis curves towards positive or negative fields, depending on the polarity of the applied voltage. At zero magnetic field, the change of voltage polarity changes the resistance value of the device (dots).

than typically $50 \mathrm{~K}$, corresponding to the occurrence of significant ME longitudinal tensor components in $\mathrm{Cr}_{2} \mathrm{O}_{3}$ [18]. Applying an external magnetic field destroys the effect, indicating the magnetic nature of the phenomenon, and suggesting that the magnetic configurations modified by the applied voltage are zero-field-cooled metastable states. The lack 


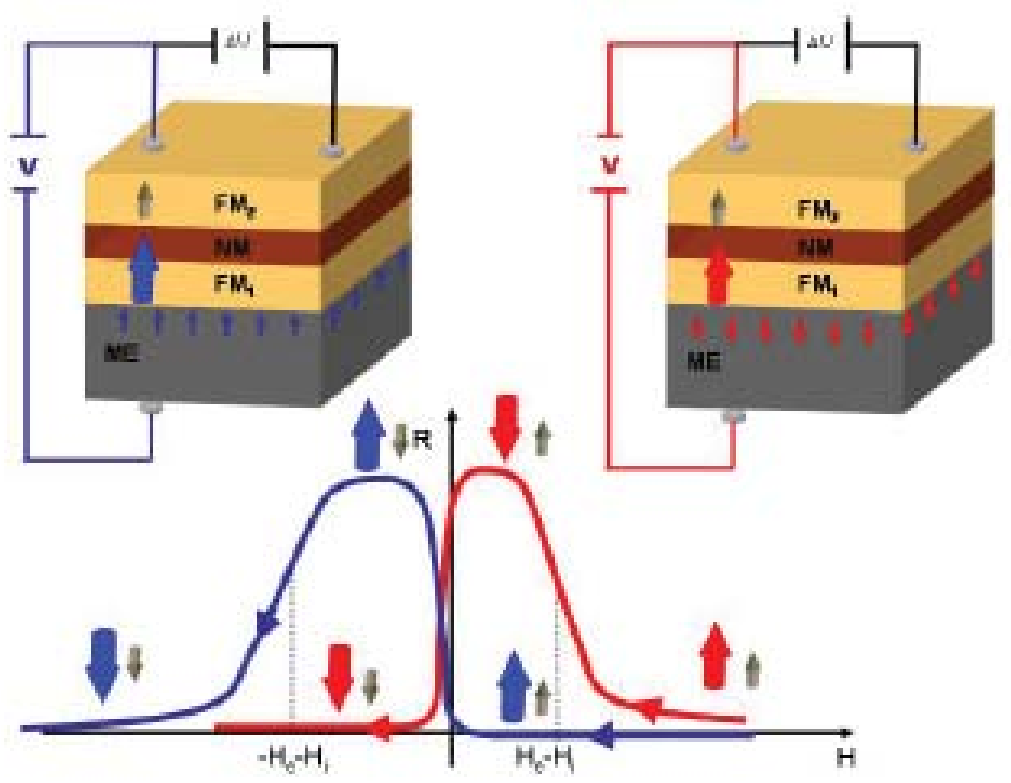

Figure 3. Schematics of the magnetoresistance curve of a GMR device involving an $\mathrm{ME}$ film as a pinning layer. The bias voltage $\Delta \mathrm{U}$ measures the resistance of the device in a current-in-plane geometry. Half-hysteresis curves are shown, after saturation at positive field values. The change of polarity of the ME layer voltage $\mathrm{V}$ changes the direction of the net magnetization of the pinning field. The pinned layer FM1 switches first at large positive field (red), or second at large negative field (blue). The low field magnetic configuration is therefore either antiparallel (red) or parallel (blue), controlled by the ME voltage $\mathrm{V}$.

of control of the growth of the $\mathrm{Cr}_{2} \mathrm{O}_{3}$ films, and the presence of impurities governing the magnetoresistance properties, makes the interpretation of the data more complicated [28]. We have however strong indications that the applied voltage modifies the local magnetic configuration, in a temperature range corresponding to the existence of significant $\mathrm{ME}$ properties of $\mathrm{Cr}_{2} \mathrm{O}_{3}$.

We propose to take advantage of an antiferromagnetic ME thin film as a dielectric tunnel junction between two ferromagnetic metallic layers. A tunnel barrier is the ideal system for sustaining very high electric fields, reaching $1 \mathrm{~V} \mathrm{~nm}^{-1}$, corresponding to the bulk dielectric breakdown values previously mentioned. We expect therefore a significant net magnetization to occur in an ME barrier. The basic principle of operation of the device involves taking advantage of the exchange field between the magnetized ME layer and the two adjacent ferromagnetic films. This creates a shift of the magnetization curves of both ferromagnetic layers proportional to the magnetization in the ME layer, or the applied voltage in the device. Following figure 1, we consider a TMR device made of a soft magnetic layer FM2 and a hard (or pinned by a bottom layer, not shown in the figure) bottom layer FM1. An exchange field value of the order of the saturation field of the soft magnetic layer, i.e. of several millitesla, will provide control of the magnetization direction of the soft layer, allowing the resistance state of the device to be set by the electric field in the ME film. The half-hysteresis magnetoresistance curves of figure 1 illustrate how a change of bias voltage allows a switching between the two resistance values at zero applied magnetic field. This effect is similar to the current-induced magnetization switching, with the advantages of larger devices and larger resistance values, more suited for applications. 
Other advantages worth mentioning are that large currents are not needed, and no small lateral size scaling is necessary.

The other proposed device is based on spin valves used in GMR systems (figure 3). We consider a maximum exchange field $\mu_{0} \mathrm{H}_{\mathrm{e}}$ due to coupling between the ME layer and the bottom ferromagnetic layer. A voltage difference $\mathrm{V}$ controls the ME layer magnetization, tuning the exchange coupling with the bottom pinned layer. Voltage control of the ME layer will therefore allow switching of the bottom layer at field values between $-\mu_{0} \mathrm{H}_{\mathrm{e}}-\mu_{0} \mathrm{H}_{\mathrm{i}}$ and $\mu_{0} \mathrm{H}_{\mathrm{e}}-\mu_{0} \mathrm{H}_{\mathrm{i}}$ for the half-hysteresis shown in figure 3 (after positive saturation field), where $\mu_{0} \mathrm{H}_{\mathrm{i}}$ is the small intrinsic switching field of a free bottom layer. The exchange field $\mu_{0} \mathrm{H}_{\mathrm{e}}$ must have a magnitude corresponding to typical pinning field values of spin valve devices, i.e. several tens of millitesla. The intrinsic longitudinal ME properties necessitate a perpendicular spin valve configuration, which has been shown to be a possible alternative to standard spin valves [29]. A voltage $\Delta \mathrm{U}$ is used to characterize the magnetoresistance of the device in a current-in-plane standard geometry (a current-perpendicular-toplane geometry requires a voltage $\Delta \mathrm{U}$ applied between top and bottom ferromagnetic layers, and would correspond to a modified drawing geometry in figure 3). Inverting the voltage $\mathrm{V}$ provides a change of the pinning direction of the bottom layer. The drawing of figure 3 illustrates how the voltage $\mathrm{V}$ controls which layer will first switch its magnetization after applying a positive saturating field. The parallel or antiparallel magnetic configuration at low field depends on whether the pinned layer has already switched direction or not. For small applied magnetic fields, controlling the magnetization state of the free layer, the magnetic configuration of the device can be therefore modified by changing the polarity of $\mathrm{V}$, providing the desired electric control of the system. This device provides additional flexibility over the TMR-type device of figure 2, as the ME control voltage is distinct from the sample bias voltage. One simple application is in terms of a logic device, which is a very attractive use of magnetic storage elements, with speed, information retention, and flexibility advantages $[30,31]$. The polarity of the ME voltage provides one logical input and the direction of an external field is the other logical input, resulting in a high or low value of the resistance as logical output. For example, we assign a logical ' 0 ' to the positive ME voltage (blue curve of figure 3), and logical value ' 1 ' to the negative ME voltage (red curve of figure 3). A positive applied field is identified to a logical ' 1 ' input, and a negative applied field to a logical ' 0 '. If both inputs are ' 0 ' or ' 1 ' the resistance value is high, assigned to logical output ' 0 '. The two other logical input configurations result in a low resistance output, assigned to output ' 1 '. This example corresponds to an exclusive OR (XOR) operation. The polarity of the saturating field provides selection of the logic functionality (the example becomes an NXOR operation if the device was previously saturated at negative field values), opening the possibility of high-speed reprogrammable logic functionality [32]. A further extension of this idea involves combining currentinduced switching due to a voltage $\Delta \mathrm{U}$ in a perpendicular geometry with the ME control due to V. Simple logics using direct electric inputs only can be achieved, avoiding completely the necessity of stray magnetic field inputs.

In conclusion, spin-dependent transport devices involving resistance states controlled by applied voltages only have been presented. By taking advantage of the magnetoelectric effect occurring in an insulating antiferromagnetic film, the electric-induced magnetization provides control of the magnetic pinning in exchange biased GMR or TMR structures. Our estimates show that significant pinning fields should result from exchange coupling between magnetoelectric and ferromagnetic layers, providing a control of the magnetization state of the ferromagnet by the applied voltage through the ME film. In TMR devices, the ME film is used as a tunnel barrier and it controls the magnetization of the soft magnetic layer. ME tunnel barriers are attractive because of the intrinsic large electric field occurring in these structures. The influence of this field on the ions' positions 
and possible related magnetic properties should be kept in mind when studying currentinduced-switching properties. In GMR devices, we propose to use the ME thin film as a tunable pinning bottom layer. These devices offer new versatility for making logic and memory devices based on the change of resistance due to the change of magnetic configurations. Our proposal requires well-controlled thin crystalline structures, where the required property involves applying a large electric field, which is in significant contrast to previous devices asking for large current density capabilities.

This research was supported by the MRSEC Program of the NSF (DMR-0213808), the Office of Naval Research (N00140210610), the Keck Foundation, and the Nebraska Research Initiative.

\section{References}

[1] Grünberg P et al 1986 Phys. Rev. Lett. 572442

[2] Baibich M N et al 1988 Phys. Rev. Lett. 612472

[3] Binasch G et al 1989 Phys. Rev. B 394828

[4] Moodera J S et al 1995 Phys. Rev. Lett. 743273

[5] Ney A et al 2003 Nature $\mathbf{4 2 5} 485$

[6] Slonczewski J 1996 J. Magn. Magn. Mater. 159 L1;

Slonczewski J 1999 J. Magn. Magn. Mater. 195261

[7] Berger L 1996 Phys. Rev. B 549353

[8] Tsoi M et al 1998 Phys. Rev. Lett. 804281

[9] Wegrowe J E et al 1999 Europhys. Lett. 45626

[10] Myers E B et al 1999 Science 285867

[11] Grollier J et al 2001 Appl. Phys. Lett. 783663

[12] Urazhdin S et al 2003 Phys. Rev. Lett. 91146803

[13] Liu Y et al 2003 Appl. Phys. Lett. 822871

[14] Huai Y et al 2003 Appl. Phys. Lett. 843118

[15] Curie P 1894 J. Physique III 3393

[16] Landau L D and Lifschitz E M 1960 Electrodynamics of Continuous Media (Oxford: Pergamon)

[17] Dzyaloshinskii I E 1960 Sov. Phys. - JETP 10628

[18] Astrov D N 1960 Sov. Phys. - JETP 11 708; Astrov D N 1961 Sov. Phys. - JETP 13729

[19] O'Dell T H 1970 The Electrodynamics of Magneto-Electric Media (Amsterdam: North-Holland)

[20] Katsufuji T et al 2001 Phys. Rev. B 64104419

[21] Kimura T et al 2003 Phys. Rev. B 67180401

[22] Hur N et al 2004 Nature 429392

[23] Lottermoser T et al 2004 Nature 430541

[24] Wang J et al 2003 Science 2991719

[25] Alexander S and Shtrikman S 1966 Solid State Commun. 4 115; Hornreich R and Shtrikman S 1967 Phys. Rev. 161506

[26] Hochstrat A et al 2003 J. Magn. Magn. Mater. 272

[27] Sokolov A et al 2003 Mater. Res. Soc. Symp. Proc. 746139

[28] Sokolov A et al 2002 Europhys. Lett. 58448

[29] Garcia F 2003 J. Appl. Phys. 938397

[30] You C Y and Bader S D 2000 J. Appl. Phys. 875215

[31] Pampuch C et al 2003 Phys. Rev. Lett. 9114720

[32] Black W C and Das B 2000 J. Appl. Phys. 876674 